\title{
Produção, acúmulo e decomposição de serapilheira em três sistemas agroflorestais no Oeste do Pará
}

\author{
Cleise Rebelo Pimentel ${ }^{1 *}$ Daniela Pauletto $\circledast^{2}$ Ananda Gabrielle de Matos Rebêlo ${ }^{3}$ Adria Fernandes da \\ Silva ${ }^{2}$ Vanessa Leão Peleja ${ }^{2}$ Edwin Camacho Palomino
}

${ }^{1}$ Universidade Federal de Lavras, Departamento de Ciências Florestais, CEP 37200-900, Lavras, MG, Brasil.
${ }^{2}$ Universidade Federal do Oeste do Pará, Rua Vera Paz, s/n (Unidade Tapajós), Salé, CEP 68040-255, Santarém, PA, Brasil
${ }^{3}$ Instituto Nacional de Pesquisas da Amazônia, Av. André Araújo, 2.936, Petrópolis, CEP 69067-375, Manaus, AM, Brasil

\section{Original Article \\ *Corresponding author: paulettoflorestal@gmail.com}

Keywords:

Litter

Organic matter

Agrosystem

Palavras-chave:

Liteira

Matéria orgânica

Agrossistema

Received in

2020/05/29

Accepted on

2021/01/25

Published in

2021/06/30

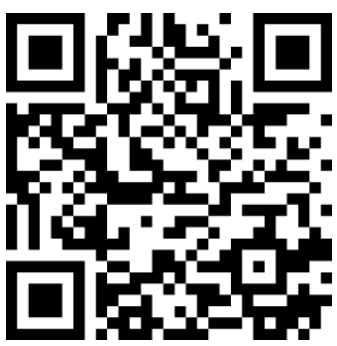

DOI: http://dx.doi.org/ 10.34062/afs.v8i1.10523

\section{(cc) BY}

RESUMO: A serapilheira é a principal via de transferência de nutrientes para o solo e estudos de produção e decomposição desse material revelam o funcionamento de ecossistemas florestais. O objetivo deste estudo foi estimar a produção e acúmulo de serapilheira, avaliar a taxa de decomposição e correlacionar o material vegetal com as variáveis climáticas em três sistemas agroflorestais no Oeste do Pará. O período de coleta foi abril a dezembro de 2017 com medições mensais. Para estimar a produção de serapilheira em cada área foram instalados seis coletores de madeira com tela de náilon $\left(0,25 \mathrm{~m}^{2}\right)$ e para o acúmulo sobre o solo foi utilizado um gabarito vazado de madeira, nas mesmas dimensões, sendo realizadas seis coletas em cada área. A maior produção total de serapilheira foi encontrada no SAF $1\left(6,24 \mathrm{t} \cdot \mathrm{ha}^{-1}\right)$, enquanto o maior estoque foi verificado no SAF $2\left(30,9 \mathrm{t} \cdot \mathrm{ha}^{-1}\right)$. A fração foliar contribuiu majoritariamente para a composição da serapilheira, exceto para o estoque do SAF 3 onde a maior contribuição foi de material lenhoso. O maior valor para a taxa de decomposição $(\mathrm{K})$ foi encontrado no SAF 1, enquanto a menor velocidade de composição para perda parcial (50 e 95\%) foi verificada no SAF 2. Velocidade do vento, umidade relativa do ar média e precipitação apresentaram correlação negativa com a serapilheira e temperatura média teve correlação positiva. Sistemas com maior diversidade de espécies apresentaram melhor taxa de decomposição e os melhores índices de produção de serapilheira.

\section{Production, accumulation and decomposition of litter in three agroforestry systems in Western Pará}

\begin{abstract}
Litter is the main route of nutrient transfer to the soil and studies of production and decomposition of this material reveal the functioning of forest ecosystems. The objective of this study was to estimate the production and accumulation of litter, to evaluate the decomposition rate and to correlate the plant material with the climatic variables in three agroforestry systems in Western Pará. The collection period was April to December 2017 with monthly measurements. In order to estimate the litter production in each area, six wooden collectors with nylon mesh $\left(0.25 \mathrm{~m}^{2}\right)$ were installed and for the accumulation on the ground, a hollow wooden template in the same dimensions was used, with six collections being made in each area. The highest total litter production was found in SAF 1 $\left(6.24 \mathrm{t} \cdot \mathrm{ha}^{-1}\right)$, while the largest stock was found in SAF $2\left(30.9 \mathrm{t} \cdot \mathrm{ha}^{-1}\right)$. The leaf fraction contributed mostly to the litter composition, except for the SAF 3 stock, where the largest contribution was made of wood material. The highest value for the decomposition rate $(\mathrm{K})$ was found in SAF 1, while the lowest speed of composition for partial loss (50 and 95\%) was found in SAF 2. Wind speed, average relative humidity and precipitation presented negative correlation with litter and average temperature had a positive correlation. Systems with greater species diversity showed a better decomposition rate and the best litter production rates.
\end{abstract}




\section{Pimentel et al}

\section{Introdução}

Os Sistemas Agroflorestais (SAFs) são sistemas de uso da terra que combinam espécies florestais, com culturas agrícolas e/ou animais, simultânea ou sequencialmente em uma mesma unidade de manejo, de acordo com um arranjo espacial e temporal, e interações ecológicas entre estes componentes, recuperando aspectos dos ecossistemas florestais como a estrutura da cobertura vegetal e a biodiversidade, restabelecendo funções ecológicas como a proteção do solo e a ciclagem de nutrientes (Nair 1984). Estes possuem grande importância em termos de sustentabilidade, no qual ao integrarem diferentes sistemas produtivos, permitem a diversificação das atividades econômicas e ambientais na área, minimizando os riscos de perdas de renda por eventos climáticos ou por outras condições adversas (Mbow et al. 2014).

A dinâmica ecológica de um ecossistema florestal é essencial para sua manutenção, onde o uso de espécies arbóreas em SAFs permitem a recuperação de áreas degradadas, por meio da redução da erosão e transferência de nutrientes para o solo. Além disso, a escolha de espécies arbóreas de crescimento rápido no início do plantio permite o aumento da disponibilidade de biomassa e promove a ciclagem de nutrientes. Essa disponibilidade de nutrientes melhoram a atividade e estrutura da fauna do solo e o equilíbrio biológico resultante auxilia no controle de pragas e doenças (Schembergue et al. 2017). A devolução se dá por meio da serapilheira e se constitui a via mais importante do ciclo biogeoquímico de nutrientes (fluxo no sistema soloplanta-solo), apresentando-se como potencial indicador de sítio (Gomes et al. 2010; Villa et al. 2016).

A serapilheira é o material presente acima do solo, constituído por folhas, material lenhoso (galhos finos, cascas e caules), material reprodutivo (flores, frutos e sementes) e outras partes da planta, bem como resto de animais e material fecal (Scoriza et al., 2012). Silva et al. 2012 afirmam que o tipo de vegetação e as variáveis ambientais, principalmente temperatura e umidade, são fatores decisivos para a quantidade de material vegetal que cai no solo, determinando a heterogeneidade e a taxa de decomposição do material depositado.

Portanto, estudos sobre a serapilheira são de fundamental importância para se estabelecer diversos critérios capazes de embasar a implantação de um SAF. Tais estudos podem informar, não somente sobre quais as espécies são mais adequadas para contribuição de serapilheira para o solo, como também fornecem informações de como o produtor deve manejar sua área a fim de mitigar possíveis problemas que venham surgir na condução do sistema.

Considerando o exposto, o objetivo do presente estudo é avaliar: (a) a eficiência dos diferentes sistemas agroflorestais no aporte e estoque de serapilheira; (b) a influência da sazonalidade; e (c) a taxa de decomposição da serapilheira em três sistemas agroflorestais na região Oeste do Pará.

\section{Material e Métodos}

Descrição da área de estudo

$\mathrm{O}$ estudo foi desenvolvido durante nove meses em três diferentes sistemas agroflorestais localizados no município de Belterra, Pará (Figura 1), no período de abril a dezembro de 2017. As condições climáticas da região, pela classificação de Koppen, são do tipo Am, altitude média de 135 m, temperatura média anual de $27,3{ }^{\circ} \mathrm{C}$, onde os maiores índices pluviométricos ocorrem entre janeiro e maio, resultando em uma precipitação anual de 1.900 a $2.200 \mathrm{~mm}$ (Alvares et al 2013). Os solos são do tipo Latossolo Amarelo com texturas médias, argilosas e muito argilosas (Ferreira 2011).

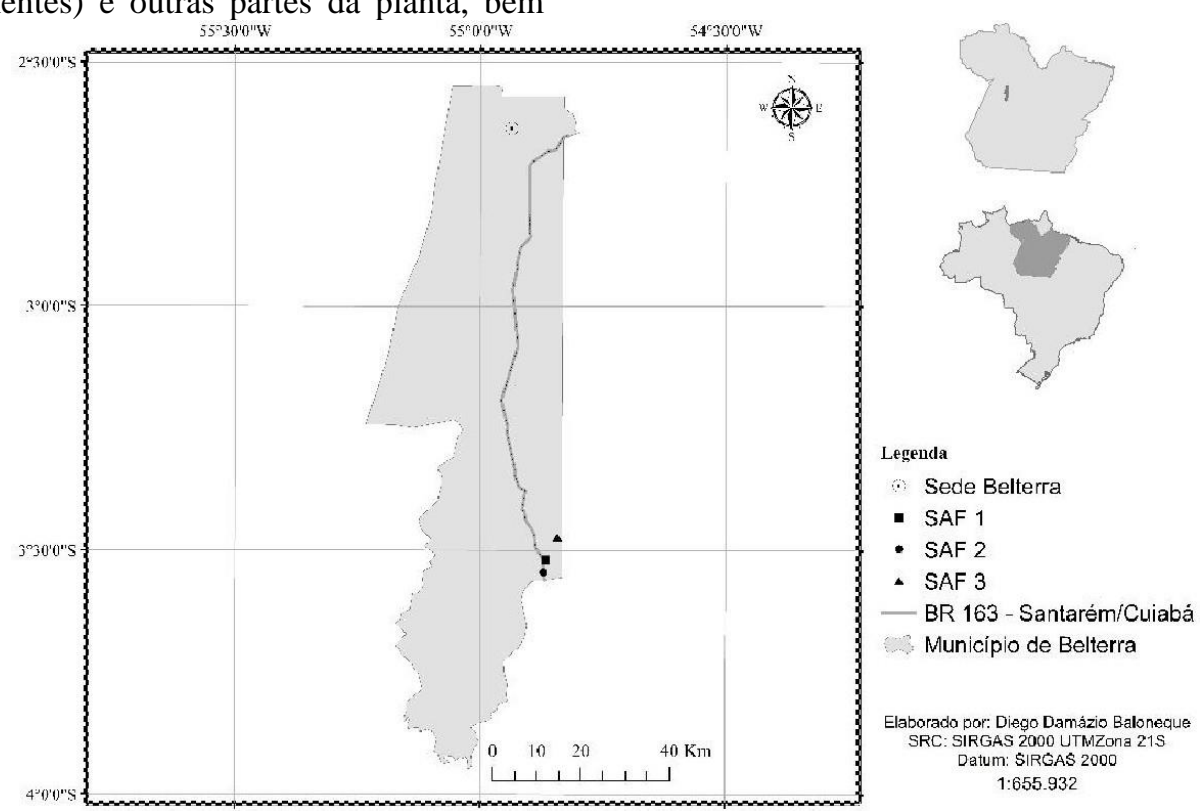

Figura 1. Localização dos três sistemas agroflorestais no município de Belterra, estado do Pará. 
Descrição dos sistemas agroflorestais

As áreas experimentais são constituídas dos seguintes arranjos: O SAF 1 está localizado nas coordenadas S $3^{\circ} 31^{\prime} 35^{\prime \prime}$ e W 54 $52^{\circ}$ '16" as margens da Rodovia Santarém-Cuiabá (BR-163), com uma área de aproximadamente 1,5 ha. É composto pelas seguintes espécies e espaçamentos: cumaru (Dipteryx spp.) (8x8 m), pimenta do reino (Piper nigrum L.) (2x2 m), banana, (Musa spp.) (4x4 m), cupuaçu (Theobroma grandiflorum (Willd. ex Spreng.) K. Schum) (4x4m), açaí (Euterpe oleracea Mart.) (4x4 m) e graviola (Annona muricata L.) (8x8 $\mathrm{m})$. Está inserido em uma área que durante cinco anos foi utilizada como pastagem. Sua implantação ocorreu em 2012 com plantios de macaxeira (Manihot spp.) nos dois primeiros anos nas entrelinhas dos plantios das perenes e semiperenes, de modo que, o cupuaçu passou a compor o sistema a partir do ano de 2015 . No período de implantação, o solo recebeu $20 \mathrm{~g}$ de adubação à base do fertilizante mineral NPK (10-28-20) e esterco de gado e galinha nas covas. O manejo da área é conduzido anualmente por meio de podas periódicas das árvores, adubação orgânica e química nas principais culturas do plantio.

O SAF 2 situa-se as margens da Rodovia Santarém-Cuiabá, nas coordenadas S $3^{\circ} 32^{\prime} 36^{\prime \prime}$ e W54 52'22”. O sistema possui um espaçamento aleatório e é composto pelas espécies taperebá (Spondias mombin L.) e pimenta do reino. Foi inserido em 2007, em uma área de regeneração natural abrangendo 0,5 hectare. Neste arranjo, a espécie taperebá tem como função ser tutor vivo para as plantas de pimenta do reino. Para a referida área, o produtor realiza roçagem periodicamente.

O SAF 3 localiza-se nas coordenadas $\mathrm{S}$ $3^{\circ} 28^{\prime} 23^{\prime \prime}$ e W 5448'17'. Constitui-se pelas espécies cumaru, pimenta do reino e moringa (Moringa oleifera L.), com espaçamento aleatório. Anteriormente, esta era uma área de pastagem, sendo utilizada para tal atividade por um período de 15 anos. O SAF foi implantado em 2012, com área de aproximadamente um hectare. O manejo deste sistema se dá pela realização de limpeza mecanizada da área, que são concentradas no período mais chuvoso do ano, podas da moringa para cobertura, adubação do solo realizadas ao longo de todo ano, adição esporádica de biofertilizantes, além da aplicação de adubação orgânica, sobretudo, em espécies com maior interesse de produção, como a pimenta do reino.

\section{Estimativa da produção e acúmulo de serapilheira}

Para estimar a quantidade de serapilheira produzida nas áreas de estudo foram instalados aleatoriamente seis coletores de madeira em cada área, com dimensões de $0,25 \mathrm{~m}^{2}$, fundo em tela de náilon com malha de $1 \times 1 \mathrm{~mm}$ e dispostos a $60 \mathrm{~cm}$ do solo. As coletas do material aportado foram realizadas mensalmente.
Para se determinar o acúmulo de serapilheira no solo, foram feitas nove coletas, com seis repetições, totalizando 18 amostras mensais. Para a realização desta coleta, fez-se o uso de um gabarito de madeira com dimensões de $0,5 \mathrm{~m}$ x $0,5 \mathrm{~m}$ o qual era lançado aleatoriamente sobre o solo e em seguida retirado o material incluso em sua área.

Após as coletas, as amostras de produção e acúmulo de serapilheira foram acondicionadas em sacos plásticos, identificadas e levadas para o Laboratório de Sementes Florestais da Universidade Federal do Oeste do Pará - UFOPA onde foram transferidas para sacos de papel, secas em estufa a $70^{\circ} \mathrm{C}$ e pesadas até obtenção de peso constante. Posteriormente, o material foi triado manualmente em fração foliar, material lenhoso (galhos e cascas), estrutura reprodutiva (flores, frutos e sementes) e material fragmentado (capim, fragmentos de adubo orgânico, fezes de animais e estruturas não identificadas). O material triado foi pesado em balança de precisão, obtendo-se assim o peso da matéria seca em gramas. Com os valores de peso seco estimou-se a deposição média de serapilheira por hectare, levando em consideração as diferentes áreas amostrais. Os dados obtidos em cada amostra foram convertidos para tonelada por hectare por meio do programa computacional Excel 2013.

\section{Estimativa da Taxa de decomposição}

Com os valores obtidos de massa seca, estimou-se a produção por hectare da serapilheira depositada nos coletores e a acumulada sobre o solo. A equação proposta por Olson (1963) e Shanks \& Olson (1961) embasou os cálculos para estimar a taxa de decomposição da serapilheira (1), a velocidade de decomposição, que é o tempo necessário para que ocorra decomposição de $50 \%$ e 95\% da serapilheira ( 2 e 3 ) e o tempo médio de renovação da serapilheira (4).

$$
\begin{gathered}
K=\frac{L}{X}(1) \\
T 0,5=\frac{-L N 0,5}{K} \\
T 0,05=\frac{3}{K}(3) \\
\frac{1}{K}(4)
\end{gathered}
$$

K é o coeficiente de decomposição, L é a produção mensal de serapilheira, $X$ é a média mensal de serapilheira acumulada, T0,5 é a velocidade de decomposição de $50 \%$ da serapilheira e T0,05 a velocidade de decomposição de $95 \%$ da serapilheira.

\section{Análise estatística}

Para avaliar a relação entre serapilheira com as variáveis climáticas foi calculada a matriz de correlação de Pearson. Os dados meteorológicos foram extraídos a partir de consulta ao banco de dados do INMET optando-se pela estação mais 
próxima das áreas de estudo localizada em Belterra (Tabela 1). Tais relações foram processadas no programa computacional Excel 2013.

Os dados foram analisados estatisticamente por meio da Análise de Variância ( $\mathrm{p}<0,05 \%$ ) e quando apresentaram diferença significativa aplicou-se o teste Tukey a $95 \%$ de probabilidade a partir do software computacional Assistat 7.7 para comparação de médias nos três sistemas agroflorestais.

Tabela 1. Variáveis climáticas da estação de Belterra no ano de 2017.

\begin{tabular}{cccccccccc}
\hline & Abr & Mai & Jun & Jul & Ago & Set & Out & Nov & Dez \\
\hline Precipitação Total & 221,80 & 116,10 & 138,90 & 42,40 & 10,40 & 68,10 & 10,00 & 0,00 & 187,00 \\
Temp. Média & 25,95 & 26,11 & 26,19 & 26,48 & 27,21 & 27,54 & 27,84 & 28,27 & 26,50 \\
Vel. Vento & 0,81 & 0,85 & 0,83 & 0,78 & 0,93 & 0,10 & 1,08 & 1,03 & 1,02 \\
UR Média & 90,22 & 89,53 & 89,38 & 87,27 & 83,59 & 81,93 & 79,67 & 79,13 & 84,85 \\
ETP Potencial & 125,00 & 130,15 & 127,2 & 136,5 & 143,86 & 150,41 & 161,95 & 168,80 & 143,24 \\
Insolação Total & 156,30 & 199,20 & 180,10 & NA & NA & 231,10 & 193,60 & 226,70 & 124,70 \\
\hline
\end{tabular}

Em que: IT - Insolação total (horas); PT - Precipitação total (mm); URM - Umidade relativa do ar média (\%); ETP

- Evapotranspiração Potencial $(\mathrm{mm})$; TM - Temperatura média $\left({ }^{\circ} \mathrm{C}\right)$; VV - velocidade do vento $\left(\mathrm{m}^{\circ} \mathrm{s}^{-1}\right)$.

\section{Resultados e discussão}

Produção de serapilheira

Para os três sistemas agroflorestais a produção total de serapilheira, para os nove meses de estudo, foi de $6,24 \mathrm{t} \cdot \mathrm{ha}^{-1}$ para o SAF 1, 4,61 th ha ${ }^{-1}$ para o SAF 2 e $1,71 \mathrm{t} \cdot \mathrm{ha}^{-1}$ para o SAF 3, com médias mensais de 0,$69 ; 0,51$ e $0,19 \mathrm{t} \cdot \mathrm{ha}^{-1}$, respectivamente. Em estudo nos mesmos sistemas Rebêlo et al (2016) encontraram valores de $8 \mathrm{t} \cdot \mathrm{ha}^{-1}$ para o SAF $1,13,5$ $\mathrm{t} \cdot \mathrm{ha} \mathrm{a}^{-1}$ para o SAF 2 e $3,7 \mathrm{t} \cdot \mathrm{ha}^{-1}$ para o SAF 3 . O sistema 1 obteve resultados aproximados de produção (nove meses) que estudo realizado por Rosa et al. 2017, no qual estimaram a produção de serapilheira durante um ano $\left(6,74 \mathrm{t} \mathrm{ha}^{-1}\right)$ em um plantio de teca com 6 anos no estado do Mato Grosso.

A Figura 2 representada os resultados das análises estatísticas para a produção mensal de serapilheira nos sistemas $(\mathrm{p}<0,05)$ onde percebe-se que a maior deposição de serapilheira ocorreu no mês de julho para as três áreas, período de transição pluviométrica, corroborando com os resultados encontrados por Villa et al. (2016), que encontraram a maior deposição de serapilheira neste mesmo período, ao estudarem o aporte de serapilheira em uma área de restauração florestal.

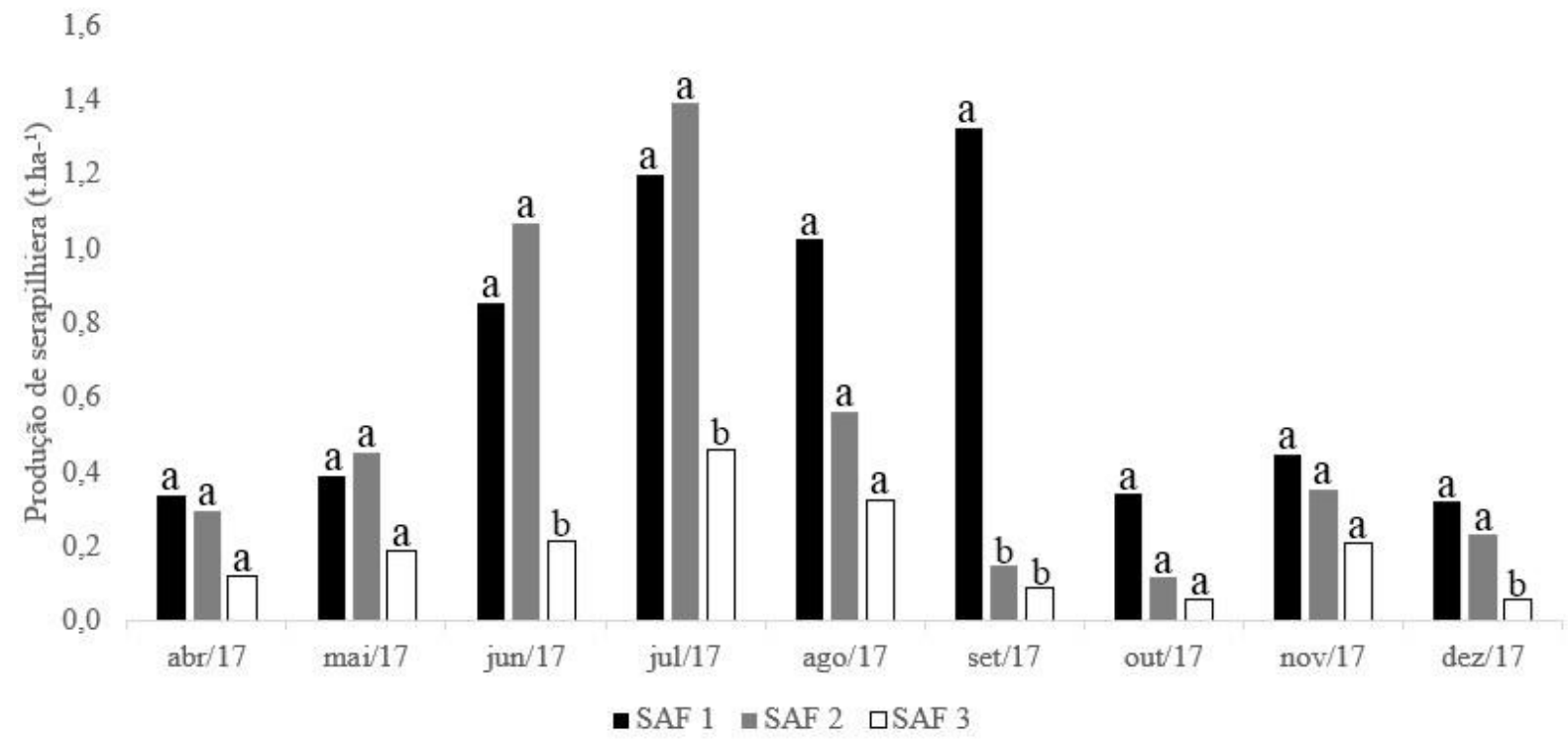

Figura 2. Produção mensal média $\left(\mathrm{t} \cdot \mathrm{ha}^{-1}\right)$ de serapilheira (abril a dezembro/2017) em sistemas agroflorestais em Belterra, Pará. Onde: letras iguais indicam que não houve diferença significativa (95\% probabilidade) entre os três sistemas no mesmo mês.

O período de maior deposição mensal de serapilheira, junho a setembro, foi diferente entre os três sistemas, que se configurou no ano de 2017 como o período de transição entre chuvoso e seco, pois nestes meses houve a precipitação de somente
$259 \mathrm{~mm}$ o que correspondeu a $32 \%$ da chuva no período do experimento. A partir dos dados do INMET (Tabela 1), para a estação de Belterra, é possível notar que neste ano não houve uma evidente estação seca, com apenas o mês de novembro sem 
registro de precipitação. Gomes et al. (2010) também verificaram que a deposição de serapilheira apresentou padrões sazonais, aportando maior quantidade de material no período de transição.

Ainda discorrendo sobre a produção de serapilheira percebe-se a ocorrência de um padrão semelhante para as três áreas quando analisados os meses de abril, maio, agosto, outubro e novembro, pois não houve diferença estatística entre os SAFs. Analisando os meses de junho e julho, nota-se uma diferença significativa entre os sistemas, no qual o SAF 3 obteve os menores valores de deposição. Isso pode ser explicado pelas espécies contidas no sistema, que possuem um diâmetro da copa pequeno e folhas de menor tamanho e peso, e no período anterior (seco) depositaram seu material vegetal para perder menos água e nutrientes. Já em setembro o SAF 1 obteve diferença significativa dos demais. $\mathrm{O}$ alto valor nesse período está associado a maior queda de material lenhoso $\left(0,31 \mathrm{t} \cdot \mathrm{ha}^{-1}\right)$, que possui um maior peso que as demais frações.

Dentre as frações avaliadas, o componente foliar foi o que mais contribuiu para a deposição de serapilheira no solo com $87,4 \%, 72,6 \%$, e $37,7 \%$ para os SAFs 2, 1 e 3, respectivamente. Isso é explicado pelo fato de as folhas possuírem uma alta taxa de transpiração, portanto, as plantas depositam uma maior quantidade de fração foliar para diminuir a perda de água. Os valores relacionados à fração foliar são aproximados aos encontrados por Pimenta et al. (2011), Santana e Souto (2011), Rosa et al. (2017) e Lorenzo e Campagnaro (2018) em estudos sobre produção de serapilheira.

O material lenhoso foi a segunda fração que mais contribuiu para a produção total da serapilheira nos sistemas com $30,3 \%, 15,4 \%$ e $10,3 \%$ para o SAF 3 , 1 e 2, respectivamente. A fração lenhosa geralmente é a segunda que mais contribui para a composição de serapilheira. Assim, os resultados encontrados corroboram com autores como Neto et al. (2015) e Villa (2016).

Os valores encontrados para material reprodutivo foram 31,0\%, 9,8\% e $1,9 \%$ respectivamente, para os SAF 3, 1 e 2. A elevada deposição de material reprodutivo no sistema 3 pode ser explicada pela presença da espécie $M$. oleifera que produz grande quantidade de flores que podem ser desprendidas com facilidade da árvore pelo vento. Já a fração de material fragmentado teve menor participação na composição da serapilheira nas três áreas estudadas (0,4 a 2,3\%).

No SAF 3, nota-se que as frações de folha, material lenhoso e reprodutivo contribuem de forma muito equilibrada para a composição da serapilheira, padrão este que não é comum de ser encontrado em outros estudos, visto que a fração folha sempre domina a composição da serapilheira. A escolha das espécies neste SAF foi determinante para esse comportamento, pois apesar da espécie $M$. oleifera perder folhas com facilidade, estas folhas não ficam no solo uma vez que o produtor as retira boa parte da copa para a comercialização e promove o amontoamento nas linhas de plantio, deixando o solo mais exposto. Outrora, a presença do material lenhoso pode apresentar alguma vantagem pelo ponto de vista nutricional, visto que galhos têm maior concentração de nutrientes diferenciada das folhas.

\section{Acúmulo de serapilheira no solo}

O acúmulo total de serapilheira no solo foi de $30,9 \mathrm{t} \cdot \mathrm{ha}^{-1}$ para o SAF 2, 19,7 t $\cdot \mathrm{ha}^{-1}$ para o SAF $1 \mathrm{e}$ $10,2 \mathrm{t} \cdot \mathrm{ha}^{1}$ para o SAF 3 e apresentou médias mensais, respectivamente, $3,4 \mathrm{t} \cdot \mathrm{ha}^{-1}, 2,2 \mathrm{t} \cdot \mathrm{ha}^{-1}$ e de $1,1 \mathrm{t} \cdot \mathrm{ha}^{-1}$. O SAF 2 destacou-se devido à grande quantidade de serapilheira estocada sobre o solo. Um dos fatores a que se atribui esses valores é o sombreamento promovido pela principal espécie do sistema (taperebá). Além disso é o sistema mais adensado quando comparado aos demais SAFs, apresentando menor espaçamento entre os indivíduos $\mathrm{e}$ consequentemente entrelaçamento de copas, favorecendo o acúmulo de material formador de serapilheira no solo. Os sistemas 1 e 3 apresentaram menores quantidades de serapilheira acumulada e tal fato justifica-se devido ao maior espaçamento e maior diversidade de espécies nestes sistemas, bem como, a adoção de tratamentos silviculturais realizados pelos produtores dessas áreas tais como roçagem, poda e capina.

Correia et al. (2016) e Villa et al. (2016) afirmam que o maior estoque de serapilheira se encontra em locais com maior fechamento de dossel o que acarreta maior desrama natural aportando maior quantidade de serapilheira no solo. Outros fatores como a baixa diversidade do consórcio e o desprovimento de práticas de manejo também podem estar relacionados ao elevado acúmulo de serapilheira (Rebêlo et al., 2016).

Os resultados das análises estatísticas para o estoque mensal de serapilheira nos sistemas $(\mathrm{p}<0,05)$ estão representadas na Figura 3. Nota-se que o SAF 2 apresenta maior estoque durante todo o período estudado. Os SAFs não apresentaram diferença significativa entre os meses de abril, maio, novembro e dezembro. Considerando os meses de junho, julho e outubro observou-se que apenas o SAF 2 diferiu-se dos SAFs 1 e 3 de forma significativa. No mês de agosto o SAF 3 apresentouse como diferente dos demais SAFs. Já no mês de setembro, apenas o SAF 3 diferiu-se dos outros SAFs.

O SAF 2 apresentou no mês de outubro o maior estoque de serapilheira durante todo o período entre os SAFs, tal comportamento já era esperado visto que este é o mês que sucede período de transição de chuvas do período estudado. Outro fator que pode ter influenciado esse estoque se deve ao fato de ser uma área de regeneração natural e adensamento elevado de árvores, tal como a 
presença da espécie caducifólia taperebá no sistema, que em período de deficiência hídrica tem como característica fisiológica a queda de suas folhas para evitar perdas de água por transpiração (Souza et al., 2013; Rebêlo et al., 2016).

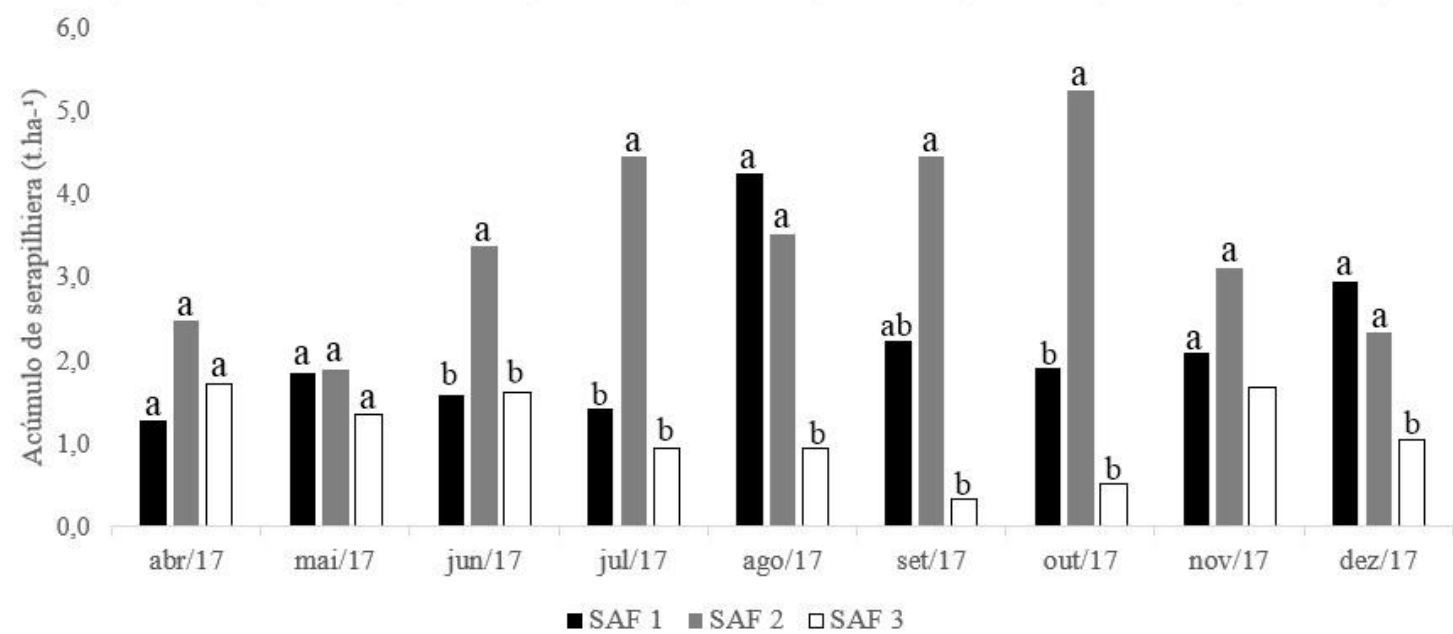

Figura 3. Acúmulo médio $\left(\mathrm{t} \cdot \mathrm{ha}^{-1}\right)$ de serapilheira no solo (abril a dezembro/2017) em sistemas agroflorestais em Belterra, Pará. Onde: letras iguais indicam que não houve diferença significativa (95\% probabilidade) entre os três sistemas dentro no mesmo mês.

Das frações que integram a serapilheira estocada no solo o material foliar foi o de maior destaque para os sistemas 1 e $2(74,2 \%$ e $54,1 \%$, respectivamente). Estes resultados se aproximam aos estudos realizados por Silva et al. (2009) e Scoriza e Piña-Rodrigues (2014) que encontraram valores de $60 \%$ e $77 \%$, para a fração foliar na serapilheira. Para o SAF 3, a fração foliar foi de $20,1 \%$ apresentandose como um valor baixo quando comparado aos resultados mais comumente encontrados pela maioria dos autores, que geralmente destacam a fração folha como a que mais contribui para a formação da serapilheira. A justificativa mais plausível para tal resultado é a retirada das folhas da M. oleifera, por parte do produtor, para a fabricação de derivados da espécie tanto para uso medicinal quanto alimentício.

As maiores frações de material lenhoso foram encontradas nos SAF $2(40,8 \%)$ e $3(37,5 \%)$. A ampla contribuição desta fração para os sistemas está relacionada ao acúmulo de galhos secos da espécie $S$. mombin que foram coletados durante o período no SAF 2. Para o SAF 3 a elevada contribuição desse material está relacionada à espécie M. oleifera que desprende com facilidade grande quantidade de galhos finos, além da poda que é realizada no sistema favorecendo o acúmulo desta fração sobre o solo. No SAF 1 foi encontrado o menor valor de participação da fração material lenhoso $(16,4 \%)$ na composição da serapilheira acumulada.

O material fragmentado foi de 7,9\% (SAF 1), $4,3 \%$ (SAF 2) e $39,8 \%$ (SAF 3) sendo que neste último a referida fração foi maior que a fração foliar. Isto pode ser explicado devido a roçagem que o produtor executa na área liberando grande quantidade de capim sobre o solo.
A fração reprodutiva foi a que menos contribuiu para a formação da serapilheira acumulada no solo durante o período observado apresentando valores de $2,6 \%, 1,6 \%$ e $0,8 \%$ para os SAFs 3, 1 e 2, nessa ordem. Isso pode ser explicado pelo fato de flores, frutos e sementes serem de fácil predação pela fauna, por serem frações mais adocicadas e de consistência com maior quantidade de água. Apesar dos baixos valores encontrados neste estudo, Santana e Souto (2011) afirmam que há uma deficiência de estudos que quantifiquem esta fração. Os mesmos autores evidenciam a necessidade de se monitorar, paralelamente, o comportamento fenológico das espécies ocorrentes nas áreas experimentais durante a fase das coletas.

\section{Estimativa da taxa de decomposição}

Na Tabela 2 são apresentadas as taxas médias mensais de decomposição (K) em cada sistema, onde tem-se os valores $2,86,1,34$ e 1,51 para os SAFs 1 , 2 e 3, respectivamente. O coeficiente $K$ é um indicador da taxa de decomposição onde quanto maior este coeficiente, mais acelerada é a velocidade de decomposição da camada de serapilheira, e consequentemente, maior liberação de nutrientes para o solo.

Para Olson (1963) são consideradas em estado de equilíbrio dinâmico as florestas tropicais cujos valores de $\mathrm{K}$ se situam entre 1,0 e 4,0. Os valores de $\mathrm{K}$ encontrados neste estudo estão dentro da faixa estabelecida pelo autor, indicando que as espécies, mesmo que em estágio de crescimento, apresentam comportamento semelhante ao de uma floresta natural. Rosa et al. (2017) analisando a decomposição em plantios de teca de 6 anos e Silva et al. (2018), em um estudo com espécies para 
recuperação florestal em plantio misto, encontraram valores de $\mathrm{K}$ inferiores ao verificado neste estudo, com médias de 0,60 e 0,47 , respectivamente.

Os resultados apontam que o coeficiente de decomposição K, é menor no SAF 2, área onde se faz presente a espécie caducifólia S. mombin, acumulando grande quantidade de folhas sobre o solo. Assim, o tempo de decomposição da serapilheira neste sistema se dará em maior espaço de tempo visto que, quanto menor o coeficiente $\mathrm{K}$, maior será o tempo para decomposição, ou seja, mais tempo residirá sobre o solo. A espécie $S$. mombin apresenta, em sua estrutura, significativos teores de celulose, hemicelulose, presença de lignina, além de ter folhas com espessura e tamanho que podem postergar o processo de decomposição (Souto et al. 2013; Lima et al. 2015).

Tabela 2. Coeficiente de decomposição (K), tempos de decomposição de $50 \%$ (T 0,5) e 95\% (T 0,05) e tempo médio de renovação $(1 / \mathrm{K})$ da serapilheira em três sistemas agroflorestais, no Oeste do Pará.

\begin{tabular}{ccccc}
\hline Sistemas & $\begin{array}{c}\text { Coeficiente de } \\
\text { Decomposição }(\mathbf{K})\end{array}$ & T 0,5 & $\begin{array}{c}\text { T 0,05 } \\
\text { (anos) }\end{array}$ & 1/K \\
\hline SAF 1 & 2,86 & 0,24 & 1,05 & 0,35 \\
SAF 2 & 1,34 & 0,52 & 2,23 & 0,74 \\
SAF 3 & 1,51 & 0,46 & 1,98 & 0,66 \\
\hline
\end{tabular}

O sistema com menor velocidade de decomposição foi o SAF 2, onde o tempo aproximadamente necessário para o desaparecimento de 50 e $95 \%$ da serapilheira foi de 0,52 e 2,23 anos mostrando que a decomposição ocorrerá de forma mais lenta considerando que o tempo médio de renovação $(1 / \mathrm{k})$ foi de 0,74 anos.

O SAF 1 apresentou maior coeficiente de decomposição $(\mathrm{K})$ indicando que neste sistema o tempo para decomposição da serapilheira é menor quando comparado aos SAFs 2 e 3. Uma provável explicação para este resultado é o espaçamento entre os indivíduos presentes no sistema, que permite uma passagem de grande quantidade de luz atingindo diretamente o solo que, aliada às condições de temperatura e umidade criam um microclima que pode facilitar o processo de decomposição do material presente no solo. Outros fatores que são possíveis explicações para o resultado encontrado são as práticas de manejo que o produtor emprega na área tais com roçagem, poda e adubação com esterco bovino que estimula a atividade microbiana no solo conferindo maior rapidez à velocidade de decomposição da matéria orgânica.

Na Figura 4, observa-se o comportamento do coeficiente de decomposição $\mathrm{K}$ ao longo do período estudado. Nota-se que o SAF 1 apresenta um pico de decomposição no mês de julho, isto pode ser explicado pelas condições ambientais favoráveis à decomposição, visto que neste período há redução da precipitação. Assim, o sistema conta com umidade e temperatura ideais para o processo de decomposição da matéria orgânica disponibilizando grande quantidade de nutrientes para o solo. Outro fator que pode ter influenciado esse comportamento foi a diversidade de espécies dentro sistemas, pois, segundo Menezes et al. (2010) a alta diversidade de espécies florestais influencia positivamente a atividade da comunidade decompositora em um ecossistema.

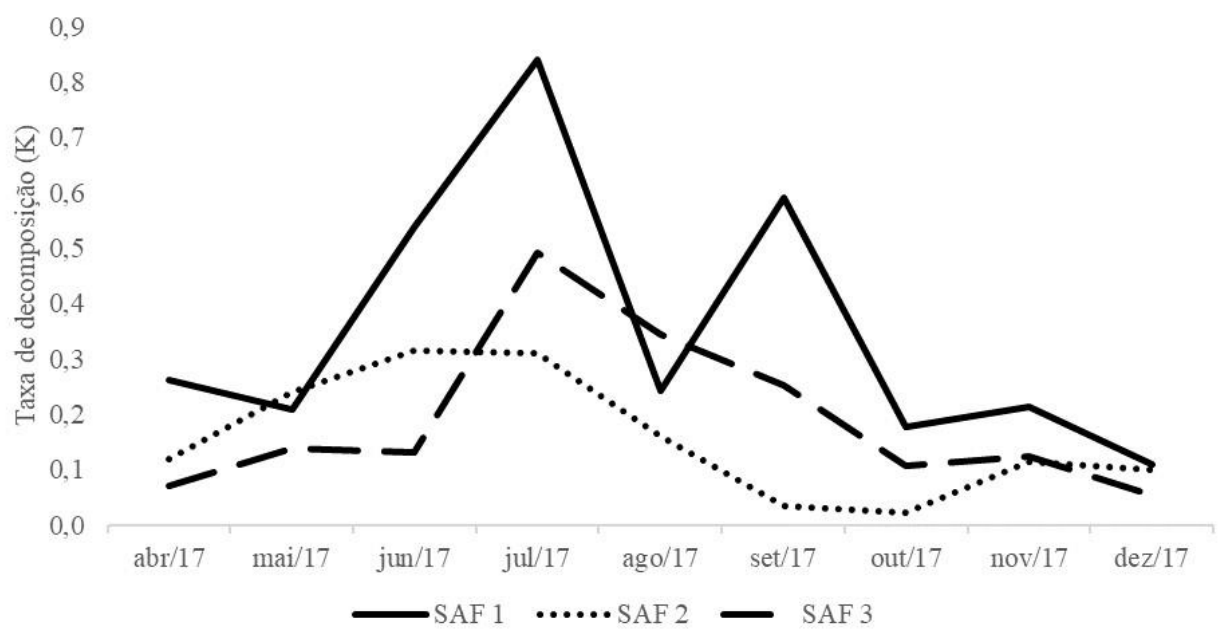

Figura 4: Comportamento do coeficiente de decomposição (K) ao longo de nove meses em três sistemas agroflorestais no Oeste do Pará 
Os sistemas 2 e 3 comportaram-se de maneira constante em relação à decomposição. Este comportamento no SAF 2 pode ser explicado pelas condições que o local apresenta: pouca entrada de luz, entrelaçamento de copas e maior camada de serapilheira sobre o solo. Já o SAF 3 apresenta-se com boa entrada de luz e espaçamento, porém, não possui alta diversidade de espécies que possam melhorar a decomposição da matéria orgânica da área.
Correlação entre produção de serapilheira $e$ variáveis climáticas

A correlação de Pearson indicou que para o período estudado, algumas variáveis climáticas podem ter influenciado no comportamento da produção e acúmulo da serapilheira nos três sistemas. Para a discussão deste estudo foram adotados somente os resultados a partir de $\mathrm{R}=0,6$, que é considerado correlação moderada. Conforme a Tabela 3 a correlação foi baixa para a maioria das variáveis.

Tabela 3. Correlação de Pearson entre variáveis climáticas e serapilheira para o período estudado nas três áreas.

\begin{tabular}{ccccccc}
\hline \multicolumn{7}{c}{ SAF 1 } \\
\hline \multirow{2}{*}{ Produção } & IT & PT & URM & ETP & TM & VV \\
Estoque & 0,5 & $-0,3$ & 0,1 & $-0,2$ & 0 & $-0,6$ \\
& 0,2 & $-0,4$ & $-0,6$ & 0,5 & 0,5 & 0,0 \\
& \multicolumn{7}{c}{ SAF 2 } \\
Produção & $-0,1$ & $-0,1$ & 0,5 & $-0,4$ & $-0,4$ & $-0,7$ \\
Estoque & 0,5 & $-0,6$ & $-0,5$ & 0,5 & 0,6 & $-0,2$ \\
& \multicolumn{7}{c}{ SAF 3 } & & & \\
& IT & PT & URM & ETP & TM & VV \\
Produção & 0,4 & $-0,4$ & 0,2 & $-0,2$ & $-0,2$ & $-0,6$ \\
Estoque & $-0,2$ & 0,4 & 0,5 & $-0,4$ & $-0,4$ & 0,4
\end{tabular}

Em que: IT - Insolação total; PT - Precipitação total; URM - Umidade relativa média; ETP - Evapotranspiração Potencial - TM - Temperatura média; VV - velocidade do vento

No SAF 1, a umidade relativa apresentou correlação negativa moderada $(r=-0,6)$ com $o$ estoque de serapilheira indicando que quanto maior a umidade relativa, menor será o estoque. Outra variável que apresentou mesmo índice de correlação que a umidade dentro deste sistema foi a velocidade do vento.

No SAF 2 a variável que apresentou correlação negativa moderada $(\mathrm{r}=-0,6)$ com $\mathrm{o}$ estoque foi a precipitação. Com isso, entende-se que quanto maior a quantidade de chuva sobre a área menor será o estoque uma vez que a umidade aumenta e acelera o processo de decomposição. Ferreira et al. (2015) avaliando uma floresta no estado do Pará também verificaram que nos maiores períodos de precipitação houve menor queda de serapilheira sobre o solo. A temperatura média apresentou correlação moderada positiva $(r=0,6)$ com o estoque de serapilheira mostrando que as árvores perdem mais folhas quando a temperatura encontra-se mais elevada.

A velocidade do vento apresentou correlação negativa forte e moderada com a produção de serapilheira $(\mathrm{r}=-0,7)$ para o SAF 2 e para os SAFs 1 e $3(r=-0,6)$. Assim, pode-se dizer que a velocidade do vento não é suficiente para interferir de forma significativa na produção de serapilheira. Os resultados de velocidade do vento encontrados no presente estudo contestam os resultados encontrados por Siqueira et al. (2016) ao estudarem a influência das variáveis climáticas na produção de serapilheira em um cerradão no estado de Minas Gerais.

\section{Conclusão}

A maior quantidade de serapilheira produzida foi encontrada no SAF 1, enquanto o maior acúmulo foi verificado no SAF 2. O período sazonal foi similar na maioria dos meses avaliados para $\mathrm{o}$ acúmulo de serapilheira, no entanto, a maior quantidade de aporte do material vegetal ocorreu no período de transição.

A fração foliar contribuiu de forma majoritária para a composição da serapilheira nos sistemas avaliados, porém as demais frações variaram conforme o sistema, tendo material lenhoso e reprodutivo especial destaque no material depositado no SAF 3.

Os sistemas com maior diversidade de espécies apresentaram maior taxa de decomposição e os melhores índices de produção de serapilheira. Os valores de $\mathrm{K}$ acima de 1 indicam que o material produzido é sempre superior ao acumulado na serapilheira e as espécies inseridas possuem alta velocidade de decomposição.

As condições climáticas pouco influenciam na produção e acúmulo de serapilheira, com exceção da precipitação, temperatura média, umidade relativa e velocidade do vento para alguns dos sistemas avaliados.

O monitoramento da produção e estoque de serapilheira se revelou uma importante ferramenta 
para inferir sobre a velocidade de decomposição da principal entrada de nutrientes nos sistemas o que, por sua vez, pode inferir sobre a dinâmica de introdução ou retirada de espécies nos plantios.

\section{Agradecimentos}

A Universidade Federal do Oeste do Pará UFOPA e ao Centro de Estudos em Manejo de Sistemas Florestais Integrados - CEMI. Aos agricultores familiares que abriram as portas de suas propriedades para a realização da pesquisa.

\section{Referências}

Alvares CA, Stape JL, Sentelhas PC, Gonçalves, JLM, Sparovek, G (2013) Koppen's climate classification map for Brazil. Zeitschrift Meteorologische Vol. 22(6): p. 711 - 728.

Correia GGS, Martins SV, Neto AM, Almeida SK (2016) Estoque de serapilheira em floresta em restauração e em floresta Atlântica de Tabuleiro no sudeste brasileiro. Revista Árvore, 40(1): 13-20. doi: 10.1590/0100-67622016000100002.

Ferreira JD (2011) Análise do plano-processo na urbanização de cidades no Baixo Amazonas: o caso de Santarém- Brasil. Dissertação, Universidade da Amazônia. 118p.

Ferreira LS, Cattânio JH, Jardim MAG (2015) Efeito da topografia e da precipitação na florística e na produção de liteira em Caxiuanã, Pará. Revista Árvore, 39(6):995-1005. doi: 10.1590/010067622015000600002 .

Gomes JM, Pereira MG, Piña-Rodrigues FCM, Pereira GHA, Gondim FR, Silva EMR (2010) Aporte de serapilheira e de nutrientes em fragmentos florestais da Mata Atlântica, RJ. Rev. Bras. Ciênc. Agrár. Recife, 5(3):383-391. doi: 10.5039/agraria.v5i3a552.

Lima RP, Fernandes MM, Fernandes MRM, Matricardi EAT (2015) Aporte e decomposição da serapilheira na Caatinga no Sul do Piauí. Floresta e Ambiente, 22(1):42-9. doi: 10.1590/21798087.062013 .

Lorenzo L; Campagnaro VH (2018) Litterfall production as a function of planting seedlings system in a two years forest restoration area in the coastalplain of Caraguatatuba, São Paulo, Brazil. Revista Árvore, 41(3): e410319. doi: 10.1590/180690882017000300019.

Mbow C, Smith P, Skole D, Duguma L, Bustamante M (2014). Achieving mitigation and adaptation to climate change through sustainable agroforestry practices in Africa. Current Opinion in
Environmental Sustainability, 6(1): 8-14. doi: 1016/j.cosust.2013.09.002.

Menezes CEG, Pereira MG, Correia MEF, Anjos LHC, Paula RR, de Souza ME (2010) Aporte e decomposição da serapilheira e produção de biomassa radicular em florestas com diferentes estágios sucessionais em Pinheiral, RJ. Ciência Florestal, 20(3): 439-452. doi: $10.5902 / 198050982059$.

Nair, PKR (1984) Tropical agroforestry systems and practices. In: Furtado JI, Ruddle K (ed) Tropical resource ecology and development. Inglaterra: John Willey Ed. Chichester. p. 39 p.

Neto APS, Barreto PAB, Gama-Rodrigues EF, Novaes AB, de Paula A (2015) Produção de serapilheira em floresta estacional semidecidual e em plantios de Pterogyne nitens tul. e Eucalyptus urophylla ST Blake no sudoeste da Bahia. Ciência Florestal, 25(3): 633-643. doi: $10.5902 / 1980509819614$.

Olson JS (1963) Energy storage and the balance of producers in ecological systems. Ecology, 44(2): 322-331. doi: 10.2307/1932179.

Pimenta JA, Rossi LB, Torezan JMD, Cavalheiro AL, Bianchini E (2011) Produção de serapilheira e ciclagem de nutrientes de um reflorestamento e de uma floresta estacional semidecidual no sul do Brasil. Acta Botânica Brasilica, 25(1):53-57. doi: 10.1590/S0102-33062011000100008.

Rebêlo AGM, Capucho HLV, Pauletto D, Neto VP (2016) Acúmulo de serapilheira em três sistemas agroflorestais no Oeste do Pará. In X Congresso Brasileiro de Sistemas Agroflorestais, Cuiabá, Mato Grosso.

Rosa TFD, Scaramuzza WLMP, Feitosa IP, Abreu FFM (2017) Produção e decomposição de serapilheira em povoamentos de teca no estado de Mato Grosso, Brasil. Ciência Florestal, 27(4): $1117-$ 1127. doi: 10.5902/1980509830288.

Santana JAS, Souto JS (2011) Produção de serapilheira na Caatinga da região semiárida do Rio Grande do Norte, Brasil. Revista IDESIA, 29(2):8794. doi: 10.4067/S0718-34292011000200011.

Schembergue A, Cunha DA, Carlos SM, Pires MV, Faria RM (2017) Sistemas Agroflorestais como Estratégia de Adaptação aos Desafios das Mudanças Climáticas no Brasil. Revista de Economia $e$ Sociologia Rural, 55(1): 9-30. doi: 10.1590/123456781806-94790550101. 
Scoriza RN, Pereira MG, Pereira GHA, Machado DL, Silva ER (2012) Métodos para coleta e análise de serrapilheira aplicados à ciclagem de nutrientes. Floresta e Ambiente, 2(2): 1-18.

Scoriza RN, Piña-Rodrigues FCM (2014) Influência da precipitação e temperatura do ar na produção de serapilheira em trecho de Floresta Estacional em Sorocaba, SP. Floresta, 44(4): 687-696. doi: 10.5380/rf.v44i4.34274.

Shanks R, Olson, JS (1961) First year breakdown of leaf litter in Southern Appalachia. Forest Science, 34(3473): 194-195. doi: 10.1126/science.134.3473.194.

Silva AD, Junior RCO, Tanabe CS, Martins ICT (2012) Influência da precipitação e temperatura do ar na produção de liteira na Floresta Nacional do Tapajós, Belterra - PA. In: XII Salão de Pesquisa e Iniciação Cientifica do CEULS/ULBRA e Conexão de saberes pela pesquisa, 2007, Santarém.

Silva BEC, Silva MRJ, Medina EF (2018) Aporte e decomposição de serapilheira em área pósmineração em processo de recuperação com espécies florestais. Revista Agrogeoambiental, 10(2):35-4. doi: 10.18406/2316-1817v10n220181098.

Silva CD, Lobo FDA, Bleich ME, Sanches L (2009) Contribuição de folhas na formação da serrapilheira e no retorno de nutrientes em floresta de transição no norte de Mato Grosso. Acta amazônica, 39(3): 591600. doi: 10.1590/S0044-59672009000300014.

Siqueira TM, Pinheiro MHO, Silva DG, Franco TM (2016) Influências climáticas na produção de serapilheira em um cerradão em Prata - MG. Biotemas, 29(2):7-15. doi: 10.5007/21757925.2016v29n2p7.

Souto PS, Souto JS, Santos RV, Bakke IA, Sales FCV, Souza BV (2013) Taxa de decomposição da serapilheira e atividade microbiana em área de caatinga. Cerne, 19(4), 559-565. doi: 10.1590/S0104-77602013000400005.

Souza AP, Mota LL, Zamadei T, Martin CC, Almeida FR, Paulino J (2013) Classificação Climática e Balanço Hídrico Climatológico no Estado de Mato Grosso. Nativa, 1(1): 34-43. doi: 10.14583/2318-7670.v01n01a07.

Villa EB, Pereira MG, Alonso JM, Beutler SJ, Leles PSDS (2016) Aporte de serapilheira e nutrientes em área de restauração florestal com diferentes espaçamentos de plantio. Floresta e Ambiente, 23(1): 90-99. doi: 10.1590/2179-8087.067513. 